\title{
From Passive to Covert Security at Low Cost
}

\author{
Ivan Damgård, Martin Geisler, and Jesper Buus Nielsen \\ Dept. of Computer Science, University of Aarhus, Denmark \\ $\{$ ivan, mg, jbn $\} @ c s . a u . d k$
}

\begin{abstract}
Aumann and Lindel defined security against covert attacks, where the adversary is malicious, but is only caught cheating with a certain probability. The idea is that in many real-world cases, a large probability of being caught is sufficient to prevent the adversary from trying to cheat. In this paper, we show how to compile a passively secure protocol for honest majority into one that is secure against covert attacks, again for honest majority and catches cheating with probability $1 / 4$. The cost of the modified protocol is essentially twice that of the original plus an overhead that only depends on the number of inputs.
\end{abstract}

\section{Introduction}

When studying cryptographic protocols, the behavior of the adversary has traditionally been categorized as being either semi-honest (passive) or malicious (active). A semi-honest adversary will only listen in on the network communication and spy passively on the internal state of the corrupted protocol participants. At the other end of the spectrum, a malicious adversary can make corrupted parties behave arbitrarily and will try to actively disrupt the computation in order to gain extra information and/or cause incorrect results.

Aumann and Lindell [2] introduce a third type of adversary called a covert adversary. This is intuitively an adversary which is able to do an active attack, but will behave correctly if the risk of being caught is sufficiently large - even if that probability is not essentially 1 . The argument for studying covert adversaries is that there are many real world situations where the consequences of being caught out-weights the benefit of cheating - even a small but non-negligible risk of being caught is a deterrent. An example could be companies that agree to conduct an auction using secure multiparty computation. If a company is found to be cheating it may be subject to fines and it will hurt its long-term relationships with customers and other companies.

In the standard simulation-based definition of secure multiparty computation a protocol is said to securely evaluate a function $f$ if no attack against the protocol can do better than an attack on an ideal process where an ideal functionality evaluates $f$ and hands the result to the parties. Aumann and Lindell [2 give three different models of what a covert adversary can do by defining two different ideal functionalities that may compute $f$ as usual, but may also act differently, depending on what the adversary does. They also define what it

The original version of this chapter was revised: The copyright line was incorrect. This has been corrected. The Erratum to this chapter is available at DOI: 10.1007/978-3-642-11799-2_36

D. Micciancio (Ed.): TCC 2010, LNCS 5978, pp. 128-145, 2010.

(C) Springer-Verlag Berlin Heidelberg 2010 
means for a protocol to implement an ideal functionality securely, this is a fairly standard simulation-based definition for sequentially composable protocols.

Thus, the special ingredient in the model that allows to accommodate covert attacks is only in the definition of the functionalities, which correspond to different levels of security, which are called Explicit Cheat Formulation (ECF) and Strong Explicit Cheat Formulation (SECF) 1 The basic idea in both cases is that the adversary may decide to try to cheat and must inform the functionality about this. The functionality then decides if the cheating is detected which happens with probability $\varepsilon$, where $\varepsilon$ is called the deterrence factor. In this case all parties are informed that some specific corrupt party cheated. Otherwise, with probability $1-\varepsilon$, the cheating is undetected, and there is no security guarantee anymore: the functionality gives all inputs to the adversary and lets him decide the outputs. The difference between the two variants is that for ECF, the adversary gets the inputs of honest parties and decides their outputs immediately when he decides to cheat. For SECF, this only happens if the cheat is not detected.

Thus, with ECF, the adversary is caught with probability $\varepsilon$, but will learn the honest parties' inputs even if he is caught. With SECF, he must try to cheat and succeed to learn anything he was not supposed to.

\subsection{Our Contribution}

In this paper we propose a new construction that "compiles" a passively secure protocol into a new protocol with covert security. The approach is generic, but for concreteness we describe the idea starting from the classical BGW protocol 6] for evaluating arithmetic circuits, and only give the full compiler in the full version of this paper [13].

We assume honest majority and synchronous communication with secure point-to-point channels. We also assume a poly-time adversary, as we use cryptographic tools.

The basic idea is to first use a protocol with full active security to do a small amount of computation. Here, we will prepare two sets of (secret-shared) inputs to the passively secure protocol. However, only one set of sharings contains the actual inputs, while the other-the dummy shares - contain only zeros. Initially, it is unknown which set is the dummy one. We then run the passively secure protocol on both sets of inputs until parties hold shares of the outputs, which they must commit to. Now we reveal which sharings contained dummy values, and everything concerning the dummy execution can be then made available to check that no cheating occurred here. If no cheating was detected, we open the outputs of the real execution.

The intuition is that the adversary has to decide whether to cheat without knowing which execution is the dummy one, and therefore we can catch him with probability $\frac{1}{2}$ if he cheats at all, so one would expect this to give a deterrence factor of $\frac{1}{2}$.

${ }^{1}$ They also have a so called Failed Simulation definition which is weaker and which we do not use here. 
However, while the intuition is straightforward, there are several non-trivial technicalities to take care of to make this work. We need parties to be able to prove that they really sent/received a given message earlier, and we have to do the final check without introducing too much overhead. After solving these problems, we obtain a protocol with deterrence factor $\frac{1}{4}$ whose complexity is essentially twice that of the passive protocol plus the overhead involved in preparing the inputs (which does not depend on the size of the computation).

It should be noted that there is an overhead involved in proving what messages were sent in the past. For this, players need to sign the messages they send. However, unless the arithmetic circuit we compute has very large depth and small breath, the cost of signing can be amortized over several operations requiring communication, and so is not significant. For the most advanced version of our construction, players also need to UC commit at the end to the set of messages they sent to each player. Our solution to this in the standard model is based on Paillier encryption and is quite elaborate, but for a practical implementation one can use the random oracle model, in which case commitment reduces essentially to hashing the messages, and is not a major cost.

We note that we focus on the complexity we get when there is no deviation from the protocol. In our construction, the adversary can slow things down by a factor linear in the number of parties by deviating, but the protocol is still secure, the adversary can only make it fail if he runs the risk of actually cheating and hence of being caught. Now, the spirit of covert security is that the adversary is to some extent rational, he does not cheat because it does not pay off to do so. It seems to us that there is little benefit in practice for the adversary in only slowing things down, while he cannot learn extra information or influence the result. We therefore believe that the complexity in practice can be expected to be what we get when there is no deviation.

We show our protocol is secure by showing that it implements Aumann and Lindell's functionality in the UC model 9], i.e., we do not use their simulation notion. The only difference this makes is that we get a stronger composition property for our protocol.

We show that the classical passively secure protocol by Ben-Or et al. 6] can be compiled to give a protocol with SECF security. Our approach can be used in a more general way, to compile any passively secure protocols into a covert protocol, if the original protocol satisfies certain reasonable conditions. The conditions are essentially as follows. The protocol should be based on secret sharing and consist of a computation phase and a reconstruction phase.

Computation phase: The computation phase starts from sharings of the inputs and produces sharings of the outputs, where the view of $t<n / 2$ passively corrupted parties is independent of the inputs being computed on.

Reconstruction phase: The reconstruction phase consists of a single message from each party to each other party-i.e., it is non-interactive.

Passive security: Suppose uniformly random sharings of the inputs are dealt by an ideal functionality. Consider the protocol that executes the computation phase on these sharings followed by the reconstruction phase. This 
protocol should be passively secure against $t<n / 2$ statically corrupted parties.

The approach to obtaining covert security is basically the same as described above. The details are described in the full version [13]. If the computation phase leaks no information, even under active attacks (as is the case for the BGW protocol), we get SECF security, otherwise ECF security is obtained.

\subsection{Related Work and Discussion}

Goyal et al. 15] improve Aumann and Lindell's 2-party protocol and also give a general multiparty computation protocol with covert security for the case of dishonest majority.

Our work focuses instead on honest majority. The skeptical reader may ask whether this is really interesting: the motivation for covert security is to settle for less than full robustness in return for more efficient protocols, and it may seem that we already know how to have great efficiency with honest majority and full active security. For instance, in [5, 10], it is shown that unconditionally secure evaluation of a circuit $\mathcal{C}$ for $n$ parties and $t<n / 3$ corruptions can be done in complexity $O(|\mathcal{C}| n)$ plus an overhead that only depends on the depth of the circuit, and in 12], it is shown under a computational assumption that this can be reduced to $O(|\mathcal{C}|)$ except for logarithmic factors plus an overhead that is independent of the circuit. Here, the security threshold can be selected arbitrarily close to $\frac{1}{2}$.

How could we hope to be better than that? There are two answers to this: First, the previous protocols are not as efficient as it may seem: the result from [12] only works asymptotically for a large number of parties and very large computations, it makes non black-box use of a pseudo-random function and is, in fact, very far from being practical. The protocols in 5, 10] use only cheap information theoretic primitives, but the security threshold in non-optimal and there is an overhead implying that deep circuits are expensive.

However, these protocols can all become much simpler and more practical if we assume the adversary is passive. For instance, when the adversary is passive the protocols from [5, 10] can tolerate $t<n / 2$ and no longer have an overhead that depends on the circuit depth. Our compiler works for any "reasonable" protocol that is based on secret sharing, so we can use it on these simpler passively secure protocols and get a protocol with covert security, but with efficiency and security threshold similar to the passively secure solutions.

The second answer is that general circuit evaluation is not the only application. There are many special purpose protocols that are designed for a passive adversary but where obtaining active security comes at a significant cost. One example is the protocol by Algesheimer et al. 1] for distributed RSA key generation. Another is the auction application described in [8]. In both cases the protocols do not go via evaluation of a circuit for the desired function, but gets significant optimizations by taking other approaches. We can use our construction here to get covert security at a cost essentially a factor of two. 


\section{Preliminaries}

Aumann and Lindell 2] present three successively stronger notions of security in the presence of covert adversaries, of which we consider the two strongest ones. There the adversary is forced to decide whether to cheat without knowledge of the honest parties' inputs. As mentioned, these are called ECF and SECF and are defined by specifying two (very similar) ideal functionalities.

For convenience, we give the ECF and SECF functionalities here. The only differences from 2] is that we do not include an option for the adversary to abort the protocol, and also, if no cheating is detected, the adversary cannot stop the functionality from giving outputs to the honest parties. This gives a stronger notion of security, and we can obtain it as we assume an honest majority.

Another difference is that we relax the requirements on the detection mechanism slightly. In [2] it is required that only one corrupted party is detected and that the honest parties agree on that party. We allow that several corrupted parties are detected and allow that different honest parties detect different sets of corrupted parties. The only requirement is that there is at least one corrupted party which is detected by all honest parties. In the presence of an honest majority, the stronger detection requirement in 2] can then be implemented using a Byzantine agreement at the end of the protocol on who should take the blame. We prefer to see this negotiation as external to the protocol and thus allow the more relaxed detection. See Fig. 1.

The functionality $\mathcal{F}_{\text {SECF }}^{f}$ is defined exactly as $\mathcal{F}_{\text {ECF }}^{f}$, except that when the adversary sends a cheat message, the functionality does not send the inputs of

Let $f$ be a function with $n$ inputs and $n$ outputs, where $n$ is the number of parties.

The ECF functionality $\mathcal{F}_{\mathrm{ECF}}^{f}$ for function $f$ with deterrence factor $\varepsilon$ works as follows:

Inputs: Any honest party $P_{i}$ sends input $x_{i}$ to $\mathcal{F}_{\mathrm{ECF}}^{f}$, while the adversary $\mathcal{A}$ sends input on behalf of the corrupted parties.

Cheat detection: Let $C \subset\{1, \ldots, n\}$ denote the indices of the corrupted parties and let $H=\{1, \ldots, n\} \backslash C$ be the honest parties. The adversary can at any time instruct $\mathcal{F}_{\mathrm{ECF}}^{f}$ to give outputs of the form (corrupt, $j$ ) for $j \in C$ to $P_{i}$ with $i \in H$. For $i \in H$, let $J_{i} \subset C$ be the set of $j$ for which $P_{i}$ output (corrupt, $j$ ).

Attempted cheat: If $\mathcal{F}_{\mathrm{ECF}}^{f}$ receives (cheat) from $\mathcal{A}$, it will send $\left(x_{1}, \ldots, x_{n}\right)$ to $\mathcal{A}$. It then decides randomly if the cheating was detected or not:

Undetected: With probability $1-\varepsilon, \mathcal{F}_{\mathrm{ECF}}^{f}$ sends (undetected) to the adversary. Then $\mathcal{A}$ specifies for each $i \in H$ an output $y_{i}$ and $\mathcal{F}_{\mathrm{ECF}}^{f}$ outputs $y_{i}$ to $P_{i}$ for $i \in H$.

Detected: With probability $\varepsilon, \mathcal{F}_{\mathrm{ECF}}^{f}$ sends (detected) to $\mathcal{A}$. In this case $\mathcal{A}$ also gets to decide the output $y_{i}$ for $i \in H$, but must ensure that $\cap_{i \in H} J_{i} \neq \emptyset$ at the end of the execution.

Output generation: If $\mathcal{A}$ did not attempt to cheat, $\mathcal{F}_{\mathrm{ECF}}^{f}$ computes outputs $\left(y_{1}, \ldots, y_{n}\right)=f\left(x_{1}, \ldots, x_{n}\right)$ and gives $y_{i}$ to $P_{i}$.

Fig. 1. Functionality $\mathcal{F}_{\mathrm{ECF}}^{f}$ 
honest parties to the adversary. This only happens if the cheating is undetected. We can now define security:

Definition 1. Protocol $\pi$ computes $f$ with $\varepsilon$-ECF (SECF) security and threshold $t$ if it implements $\mathcal{F}_{\mathrm{ECF}}^{f}\left(\mathcal{F}_{\mathrm{SECF}}^{f}\right)$ in the $U C$ model, securely against poly-time adversaries corrupting at most $t$ parties.

This definition naturally extends to a hybrid UC model where certain functionalities are assumed to be available. By the UC composition theorem and given implementations of the auxiliary functionalities, a protocol follows that satisfy the above definition without auxiliary functionalities.

In the following, we will consider secure evaluation of an arithmetic circuit $\mathcal{C}$ over some finite field $K$. We assume that each input and output of $\mathcal{C}$ is assigned to some party, whence $\mathcal{C}$ induces in a natural way a function $f_{\mathcal{C}}$ of the form considered above. In the following, "computing $\mathcal{C}$ securely" will mean computing $f_{\mathcal{C}}$ securely in the sense of the above definition.

We will denote the participants in the protocol by $P_{1}, \ldots, P_{n}$ for a total of $n$ parties. Shamir secret sharing of $a \in K$ with threshold $t$ results in a set of shares denoted by $[a]_{t}$ or simply $[a]$ when the threshold is clear from the context. The share held by $P_{i}$ is denoted $a_{i}$.

\section{Auxiliary Functionalities}

We define some ideal functionalities to make the presentation clearer. We show how to implement them Section 5.

Message Transmission Functionality. Functionality $\mathcal{F}_{\text {TRANSMIT }}$ is an enhancement of the standard model for secure point-to-point channels. It essentially allows to prove to third parties which messages one received during the protocol, and to further transfer such revealed messages. It does not commit the corrupted parties to what they sent to each other. See Fig. 2 for details.

The ideal functionality $\mathcal{F}_{\text {TRANSmit }}$ works with message identifiers mid encoding a sender $s($ mid $) \in\{1, \ldots, n\}$ and a receiver $r($ mid $) \in\{1, \ldots, n\}$. We assume that no mid is used twice. The functionality works as follows:

Secure transmit: When receiving (transmit, mid, $m$ ) from $P_{s(m i d)}$ and receiving (transmit, mid) from all (other) honest parties, store (mid, m), mark it as undelivered, and output $(\mathrm{mid},|\mathrm{m}|)$ to the adversary. If $P_{s}$ does not input a (transmit, mid, $m$ ) message, then output (corrupt, $s($ mid $)$ ) to all parties.

Synchronous delivery: At the end of each round, deliver each undelivered $($ mid,$m)$ to $P_{r(m i d)}$ and mark $($ mid, $m)$ as delivered.

Reveal received message: On input (reveal, $m i d, i)$ from a party $P_{j}$ which at any point received the output $(\mathrm{mid}, \mathrm{m})$, output $(\mathrm{mid}, \mathrm{m})$ to $P_{i}$.

Do not commit corrupt to corrupt: If both $P_{j}$ and $P_{s}$ are corrupt, then the adversary can ask $\mathcal{F}_{\text {TRAnsmit }}$ to output $\left(\mathrm{mid}, \mathrm{m}^{\prime}\right)$ to any honest $P_{i}$ for any $m^{\prime}$ and any mid with $s($ mid $)=s$.

Fig. 2. Ideal Functionality $\mathcal{F}_{\text {TRAnSmit }}$ 
This functionality will be used for all private communication in the following, and provides a way to reliably show what was received at any earlier point in the protocol. This is used when the dummy execution is checked for consistency.

Input Functionality. For notational convenience we assume that each $P_{i}$ has one input $x_{i} \in K$. The input functionality is given in Fig. 3. Note that we let the adversary pick the dummy inputs, which is done simply not to decide at this abstract level on any specific set of dummy inputs. We also let the adversary pick the shares the functionality should produce for corrupt players. This is necessary to be able to implement the functionality with a real-life protocol.

The ideal functionality $\mathcal{F}_{\text {INPUT }}$ is parametrized by a secret sharing scheme, sss, and works as follows.

1. Receive an input $x_{i}$ from each $P_{i}$ and an input $\left(d_{1}, \ldots, d_{n}\right)$ from the adversary. The adversary also inputs $x_{i}$ for $i \in C$.

2. Flip a uniformly random bit $d \in_{\mathrm{R}}\{0,1\}$.

3. Let $e=1-d$. Let $x^{(i, d)}=d_{i}$ be the dummy inputs and let $x^{(i, e)}=x_{i}$ be the enriched inputs.

4. For every $x^{i, d}$ and $x^{i, e}$, the adversary inputs sets of shares $X^{i, d}$ and $X^{i, e}$. They each contain a share for every player in $C$, and we think of $X^{i, d}$ as the set of shares of $x^{i, d}$ that the adversary wants the functionality to produce for corrupt players.

5. For $j=1, \ldots, n$ and $c=0,1$, sample $\left[x^{(j, c)}\right] \leftarrow \operatorname{sss}\left(x^{(j, c)} \mid X^{j, c}\right)$, by which we mean that shares of $x^{i, c}$ are sampled, conditioned on players in $C$ receiving shares $X^{i, c}$.

6. Output $\left(x_{i}^{(j, 0)}\right)_{j=1}^{n}$ and $\left(x_{i}^{(j, 1)}\right)_{j=1}^{n}$ to $P_{i}$.

7. On a later input (reveal, $i, k)$, output $d$ and $\left(x_{i}^{(j, d)}\right)_{j=1}^{n}$ to $P_{k}$.

Fig. 3. Ideal Functionality $\mathcal{F}_{\text {INPUT }}$

Commitment Functionality. We use a flavor of commitment where the committer cannot avoid that a commitment is revealed. Details are in Fig. 4.

The functionality $\mathcal{F}_{\text {соммтт }}$ uses commitment identifiers encoding the sender $s($ cid $)$ of the commitment. It works as follows:

Commit: On input (commit, cid, $m$ ) from $P_{s(c i d)}$ and input (commit, cid) from all (other) honest parties, store $($ cid, $m$ ) and output (commit, cid, $|m|$ ) to the adversary.

Reveal: On input (reveal, cid, $r$ ) from all honest parties, where $(c i d, m)$ is stored, give $(c i d, m)$ to $P_{r}$.

Fig. 4. Ideal Functionality $\mathcal{F}_{\text {сомміт }}$

Coin-Flip Functionality. We use the coin-flip functionality given in Fig. $\mathbf{5}$. 
The functionality $\mathcal{F}_{\text {FLIP }}^{B}$ is parametrized by a positive integer $B$ and works as follows:

1. Sample a uniformly random $k \in \in_{\mathrm{R}}\{0, \ldots, B-1\}$.

2. When the first honest party inputs (flip), output $k$ to the adversary.

3. If in the round where the first honest party inputs ( $f l i p)$ there is some party $P_{i}$ which does not input (flip), then output (corrupt, $i$ ) to all parties.

Fig. 5. Ideal Functionality $\mathcal{F}_{\text {FLIP }}^{B}$

\section{Protocol}

Having defined the necessary ideal functionalities, we will now describe how we use them to compile the classical passively secure protocol by Ben-Or et al. [6] based on Shamir secret-sharing into one with covert security. This protocol computes an arithmetic circuit $\mathcal{C}$ with passive security. Assuming the inputs to the arithmetic circuit have been secret shared, the protocol does addition by having parties add their shares locally, and multiplication by local multiplication of shares followed by a re-sharing by each parties of the local products. Due to space constraints, we assume the details are known to the reader.

The protocols in this section use the auxiliary functionalities we defined. Thus the actual complexity of our construction depends on the implementation of those auxiliary functionalities. It turns out that the overhead incurred includes a contribution coming from the cryptographic primitives we use, this overhead does not depend on the communication complexity of the protocol we compile. In addition, the adversary can choose to slow down $\mathcal{F}_{\text {TRANSMIT }}$ by a factor of $n$, but since he cannot make it fail, a covert adversary is unlikely to make such a choice as discussed in the introduction.

We begin with a simple construction which has a rather poor computational complexity. Following that, we show how the simple protocol can be adapted to yield a better complexity.

Theorem 1. The protocol in Fig. 6 computes $\mathcal{C}$ with $\frac{1}{2}-S E C F$ security and threshold $t<n / 2$ in the $\left(\mathcal{F}_{\text {TRANSMIT }}, \mathcal{F}_{\text {INPUT }}, \mathcal{F}_{\text {COMMIT }}, \mathcal{F}_{\text {FLIP }}\right)$-hybrid world against a static adversary.

Proof. Initially $\mathcal{S}$ is given the inputs of the corrupt parties. It passes them on to $\mathcal{A}$ and simulates the protocol execution up until the point where the bit $d$ is revealed and it is determined which of the two executions were the dummy execution. $\mathcal{S}$ does this by inventing random shares whenever $\mathcal{A}$ would expect to see a share from an honest party. $\mathcal{A}$ will always see only $t$ shares and any subset of size $t$ look completely random in the real protocol execution. $\mathcal{S}$ can therefore simulate them perfectly by giving $\mathcal{A}$ random values.

During the protocol, $\mathcal{A}$ is observed by $\mathcal{S}$ and it can thus be determined if $\mathcal{A}$ ever sends an incorrect intermediate result to one of the honest parties.

- If $\mathcal{A}$ did not cheat at all, or if $\mathcal{A}$ cheated in both executions, then $\mathcal{S}$ simply follows the protocol. In the first case $\mathcal{F}_{\mathrm{SECF}}^{f_{\mathcal{C}}}$ will give $\mathcal{S}$ the outputs for the 
In general, if any of the ideal functionalities output (corrupt,j) to $P_{i}$, then $P_{i}$ also outputs (corrupt, $j$ ). Not mentioning this further, the protocol proceeds in five steps:

1. All parties provide input to $\mathcal{F}_{\text {INPUT }}$. In return they obtain shares of secret sharings $\left[x^{(j, 0)}\right]$ and $\left[x^{(j, 1)}\right]$ for $j=1, \ldots, n$. Nobody knows which sharings are dummy at this point.

2. Each party $P_{i}$ generates random keys $K_{i}^{0}$ and $K_{i}^{1}$ and commit to them using $\mathcal{F}_{\text {сомміт }}$ twice.

3. The passively secure protocol is run on both input sets $\left\{\left[x^{(j, 0)}\right]\right\}_{j=1}^{n}$ and $\left\{\left[x^{(j, 1)}\right]\right\}_{j=1}^{n}$. This evaluates the circuit $\mathcal{C}$ twice. The parties use $\mathcal{F}_{\text {сомміт to }}$ commit to their shares of the output. All randomness used in the first and second protocol run come from pseudo-random generators seeded by $K_{i}^{0}$ and $K_{i}^{1}$, respectively.

4. The parties query $\mathcal{F}_{\text {INPUT }}$ for the random bit $d$ and the shares of $\left\{\left[x^{(j, d)}\right]\right\}_{j=1}^{n}$. They then use $\mathcal{F}_{\text {соммт }}$ to reveal the key $K_{i}^{d}$ used for the pseudo-random generator for all $P_{i}$. Knowing the initial inputs and the seed for the pseudo-random generator used, the entire message trace of all parties is fixed. The parties also open the commitments to the dummy output shares.

5. Each party locally simulates the entire dummy execution to determine if any cheating took place. This amounts to checking for each party whether his input shares of $\left[x^{(j, d)}\right]$ (revealed by $\mathcal{F}_{\text {INPUT }}$ ) and seed $K_{i}^{d}$ (revealed by $\mathcal{F}_{\text {сомміт }}$ ) together lead to the shares he claims to have obtained of the output (revealed by $\left.\mathcal{F}_{\text {сомміт }}\right)$ if he follows the passively secure protocol on the messages that other parties would have sent if they followed the protocol on their shares and expanded randomness. If no discrepancies are found, the output shares of the real execution are opened.

Otherwise, the honest parties must determine who cheated

The parties have already locally simulated the dummy execution so they know the correct message trace. It is therefore simple to match this against the actual message trace revealed by $\mathcal{F}_{\text {TRAnsmit }}$ and pinpoint the first deviation. If $P_{j}$ made the first mistake, the honest parties output (corrupt, $j$ ) and halt.

${ }^{a}$ Note that it is possible for a corrupt party to "frame" an honest party by sending him wrong intermediate results. The honest party cannot tell the difference and will produce incorrect output. $\mathcal{F}_{\text {TRANSMIT }}$ is there to safeguard honest parties against this form of attack. The parties call it to reveal all messages that were received in the dummy execution.

Fig. 6. Simple version

corrupt parties, which $\mathcal{S}$ can pass along to $\mathcal{A}$ unchanged. In the second case, $\mathcal{A}$ will be caught with certainty before seeing anything which depend on the honest parties inputs. $\mathcal{S}$ can therefore simulate the protocol execution towards $\mathcal{A}$ using random shares only.

- If $\mathcal{A}$ cheats in execution $d^{\prime}$ (first or second execution), $\mathcal{S}$ will send (cheat) to $\mathcal{F}$. The functionality then determines if the cheat was successful: 
Detected: The simulator must now ensure that $\mathcal{A}$ believes he cheated in the dummy execution.

$\mathcal{A}$ will want to query $\mathcal{F}_{\text {INPUt }}$ for the value of $d$ and the shares of the dummy inputs. In response, $\mathcal{S}$ sends a response with $d=d^{\prime}$, which means that $\mathcal{A}$ cheated in the dummy execution. $\mathcal{S}$ must also send back shares of the inputs $\left\{x^{(j, d)}=d_{j}\right\}_{j=1}^{n}$ consistent with the shares $\mathcal{A}$ has already seen. At this point $\mathcal{A}$ has only seen the shares it chose for the (non-qualified) subset of corrupt parties when $\mathcal{F}_{\text {INPUT }}$ was called initially. $\mathcal{S}$ can therefore choose polynomials that agree with these values and correspond to a sharing of the inputs $d_{j}$, and finally compute consistent shares of the honest parties using these polynomials.

If $P_{j}$ were the first corrupt party who send an incorrect message to an honest party, $\mathcal{S}$ will send (corrupt, $j$ ) to $\mathcal{F}_{\mathrm{SECF}}^{f_{\mathcal{C}}}$.

Undetected: In this case the functionality responded with (undetected) together with the honest parties' inputs. The simulator must therefore make it look as if $\mathcal{A}$ cheated in the execution that was not opened, i.e., the real execution. As above, $\mathcal{S}$ can compute polynomials that will give a correct sharing of inputs based on what $\mathcal{A}$ already knows and with $d=1-d^{\prime}$.

Using these inputs together with the corrupt parties' inputs and outputs, $\mathcal{S}$ can now compute the consequence of $\mathcal{A}$ 's cheating, i.e., the altered outputs of the honest parties. It passes these outputs to $\mathcal{F}_{\text {SECF }}^{f_{\mathcal{C}}}$ as the honest parties' outputs.

It is clear that the above simulation matches the output of $\mathcal{A}$ in the hybrid world perfectly when $\mathcal{A}$ did not cheat and when $\mathcal{A}$ was foolish enough to cheat in both executions.

When $\mathcal{A}$ cheats in just one execution, $\mathcal{S}$ will make the honest parties output (corrupt, $j$ ) for some corrupt $P_{j}$ (if $\mathcal{A}$ was detected) or output normal outputs (if $\mathcal{A}$ was undetected). Each of these two cases are picked with probability exactly $\frac{1}{2}$ by the random choice made by $\mathcal{F}_{\text {SECF }}^{f_{\mathcal{C}}}$. We get the same probability distribution in the hybrid world where $\mathcal{F}_{\text {INPUT }}$ picks the bit $d$ uniformly at random.

In total, we can now conclude that the protocol in Fig. [6] computes $f_{\mathcal{C}}$ with $\frac{1}{2}$-SECF security.

The above protocol has each party execute the passively secure protocol twice after which each party simulates the actions of all other parties in the dummy execution. In the standard BGW protocol [6], each party has a computational complexity of $\mathcal{O}(n)$ per gate. By asking every party to simulate every other party, we increase the computational complexity to $\mathcal{O}\left(n^{2}\right)$ per gate.

The communication complexity is doubled by running the passively secure protocol twice. In the normal case where the dummy execution is found to contain no errors, the communication complexity is increased no further. When errors are detected, every party is sent the messages communicated by every other party. This will again introduce a quadratic blowup, now in the communication complexity. We argued in the introduction that even a small fixed probability of 
catching misbehavior is enough to deter the parties. Because of that, we expect to find no discrepancies most of the time, and thus obtain the same communication complexity as the original protocol within a constant factor. We still have a quadratic blowup in the computational complexity. However, local computations are normally considered free compared to the communication, i.e., the network is expected to be the bottleneck. So for a moderate number of parties, this simple protocol can still be quite efficient.

Still, we would like to lower the complexity when errors are detected. Below we propose a slightly more complex protocol which has only a constant overhead in both computation and communication both when no errors are detected and when the parties are forced to do a more careful verification.

This is a modification of the protocol in Fig. [6] After running Step 1-[3] unchanged, it continues with:

1. All $P_{i}$ use $\mathcal{F}_{\text {соммтт }}$ to commit to their view of the protocol, i.e., all messages exchanged between $P_{i}$ and $P_{j}$ for all $j$. This results in commitments $\operatorname{comm}_{\{i, j\}}^{(i, 0)}$ for the first execution and $\operatorname{comm}_{\{i, j\}}^{(i, 1)}$ for the second, where $\operatorname{comm}_{\{i, j\}}^{(m, c)}$ is the view of $P_{m}$ of what was sent between $P_{i}$ and $P_{j}$ in execution number $c$.

2. The parties query $\mathcal{F}_{\text {INPUT }}$ for the random bit $d$ and the shares of the dummy inputs. They then use $\mathcal{F}_{\mathrm{FLIP}}^{n-1}$ to flip a uniformly random $k \in\{1, \ldots, n-1\}$ that will be used when checking. $\mathcal{F}_{\text {Соммт }}$ is used by all parties to reveal the key $K_{i}^{d}$ used for the pseudo-random generator for all $P_{i}$. Finally, the commitments to shares in the output from the dummy execution are opened.

3. Each party $P_{i}$ checks $P_{l}$, where $l=(i-1+k \bmod n)+1$, i.e., he checks $P_{i+k}$ with wraparound from $P_{n}$ back to $P_{1}$.

The commitments $\operatorname{comm}_{\{l, j\}}^{(j, d)}$ and $\operatorname{comm}_{\{l, j\}}^{(l, d)}$ are opened to $P_{i}$, i.e., the committed views of $P_{l}$ and $P_{j}$ of what was exchanged between them. If there is a disagreement, then $P_{i}$ broadcasts a complaint and $P_{l}$ and $P_{j}$ must decommit to all parties and use $\mathcal{F}_{\text {TRAnSmit }}$ to show which messages they received from the other. This will clearly detect at least one corrupt party among $P_{l}$ and $P_{j}$ if $P_{i}$ was honest, or reveal $P_{i}$ as corrupt if the commitments were equal after all, i.e., if $P_{i}$ made a false accusation.

If all committed views agree, then $P_{i}$ simulates the local computations done by $P_{l}$ and checks whether this leads to the shares of the dummy output opened by $P_{l}$ and the messages sent according to $\operatorname{comm}_{\{l, j\}}^{(l, d)}$. If a deviation is found, $P_{i}$ broadcasts an accusation against $P_{l}$, and all parties check $P_{l}$ as $P_{i}$ did. If they verify the deviation they output (corrupt, $l$ ), otherwise they output (corrupt, $i$ ).

4. If no accusations were made, the output of the real execution is opened.

Fig. 7. Efficient version

If no errors are detected, each party does two protocol executions followed by a check of the input/output behavior of one other party. This is clearly a constant factor overhead compared to the passively secure protocol. When a party is accused, all other parties must check this party. This adds only a linear overhead to the overall protocol, and thus the protocol in Fig. [7 has a linear overhead compared to the passively secure protocol. 
It might seem as an overkill in the protocol in Fig. 7 to use $\mathcal{F}_{\text {TRAnSmit }}$ for communication and then also have the parties commit to their communication using $\mathcal{F}_{\text {Соммт }}$. The reason for the commitments is to commit the corrupted parties to what they sent among each other before it is revealed which parties check which parties. If we do not do that, they might decide on which of them was the deviator after the revelation of $d$ and $k$ and thus always pick the deviator to be one which is checked by a corrupted party. For an example of what can go wrong without the commitments the interested reader can refer to the CHINESEWHISPERS protocol in the full version of this paper [13].

Theorem 2. The protocol in Fig. 7 computes $\mathcal{C}$ with $\frac{1}{4}-S E C F$ security and threshold $t<n / 2$ in the $\left(\mathcal{F}_{\text {TRAnSmit }}, \mathcal{F}_{\text {InPUt }}, \mathcal{F}_{\text {COMmit }}, \mathcal{F}_{\text {FLIP }}\right)$-hybrid world.

Proof. The simulator for the protocol in Fig. 7 runs like the simulator for the protocol in Fig. 6. except that it must now only output (corrupt, $i$ ) to $\mathcal{F}$ if it determines that a message trace for a corrupt party $P_{i}$ was checked by an honest party, and it must do while maintaining the same probability distribution as in the hybrid world.

As before, $\mathcal{S}$ will simulate $\mathcal{A}$ and observe the messages sent to honest parties. As soon as an incorrect message is observed in execution $d^{\prime}$ and all parties committed to their communication with the other parties, we know there exists an offset $k^{\prime} \in\{1, \ldots, n-1\}$ for which an honest $P_{i}$ would catch a corrupt $P_{l}$, where $l=\left(i-1+k^{\prime} \bmod n\right)+1$ in execution $d^{\prime}$ :

- If two parties $P_{l}$ and $P_{j}$ committed to $\operatorname{comm}_{\{l, j\}}^{\left(l, d^{\prime}\right)} \neq \operatorname{comm}_{\{l, j\}}^{\left(j, d^{\prime}\right)}$, then one of them is corrupted, $P_{l}$ say, and we pick $k^{\prime}$ such that $P_{l}$ is checked by an honest $P_{i}$.

- If $\operatorname{comm}_{\{l, j\}}^{\left(l, d^{\prime}\right)}=\operatorname{comm}_{\{l, j\}}^{\left(j, d^{\prime}\right)}$ for all pairs of parties, then the wrong message sent to an honest party in execution $d^{\prime}$ implies that some party $P_{l}$ is committed to values which are not consistent with an execution of the protocol, and we pick $k^{\prime}$ to ensure that $P_{l}$ is checked by an honest party 2

The simulator sends (cheat) to $\mathcal{F}_{\mathrm{SECF}}^{f_{\mathcal{C}}}$. We have two outcomes:

Detected: Set $d=d^{\prime}$ and sample $k$ at random such that $P_{l}$ is checked by an honest party.

Undetected: Set $d=d^{\prime}$ with probability $\frac{1}{3}$, and $d=1-d^{\prime}$ otherwise. Sample $k \in\{1, \ldots, n-1\}$ such that $P_{l}$ is checked by an honest party with probability $\alpha=\frac{4}{3}\left(\frac{n-t}{n-1}-\frac{1}{4}\right)$.

If $\mathcal{A}$ did not cheat, $\mathcal{S}$ selects $d$ and $k$ as in the hybrid protocol. The simulation continues as in the hybrid world with these choices for $d$ and $k$. The ideal world output clearly match the hybrid world.

\footnotetext{
${ }^{2}$ Note that $P_{l}$ need not be the one who sent the incorrect message to the honest party $-P_{l}$ may have behaved locally consistent given its inputs-but $\mathcal{S}$ will be able to find a first deviator, and it will clearly not be one of the honest parties.
} 
When $\mathcal{A}$ did cheat, we will show that $d$ and $k$ are picked with the correct distribution. First note that $\mathcal{S}$ pick $d=d^{\prime}$ with probability $\frac{1}{4} \cdot 1+\frac{3}{4} \cdot \frac{1}{3}=\frac{1}{2}$, as in the hybrid world.

For the selection of $k$, note that a cheating party will always have a unique distance to every honest party. These distances make up a subset of $\{1, \ldots, n-1\}$ of size $n-t$. The cheater is caught exactly when the offset is picked within this subset. This happens with probability $\frac{n-t}{n-1}$ in the hybrid world. The simulator picks $k$ among the indices of honest parties with the same probability: $\frac{1}{4}+\frac{3}{4} \alpha=$ $\frac{n-t}{n-1}$. We conclude that $\mathcal{S}$ will simulate the hybrid world.

\section{Implementation of Sub-protocols}

In this section we sketch how to implement the sub-protocols described above.

Detection. In all sub-protocols we will need a tool for stopping the protocol "gracefully" when corruption is detected This is done by all parties running the following rules in parallel.

1. If a party $P_{i}$ sees that a party $P_{d}$ deviates from the protocol, then $P_{i}$ signs (corrupt, $d$ ) to get signature $\gamma_{i}$ and sends the signature to all parties. Then $P_{i}$ outputs (corrupt, $d$ ).

2. If $P_{k}$ received a signature $\gamma_{i}$ on (corrupt, $d$ ) from $t+1$ distinct parties $P_{i}$, it considers these as a proof that $P_{d}$ is corrupted, sends this proof to all parties, outputs (corrupt, $d$ ), waits for one round and then terminates all protocols.

3. If $P_{k}$ receives a proof that $P_{d}$ is corrupt from any party, it relays this proof to all parties, outputs (corrupt, $d$ ), waits for one round and then terminates all protocols.

If the signature scheme are unforgeable and only corrupted parties deviate from the protocol, then the protocol has the following two properties, except with negligible probability.

Detection soundness: If an honest party outputs (corrupt, $d$ ), then $P_{d}$ is corrupt.

Common detection: If an honest party terminates the protocol prematurely, then there exists $P_{d}$ such that all honest parties have output (corrupt, $d$ ).

The reason why the relayer $P_{r}$ waits for one round before terminating is that $P_{r}$ wants all other parties to have seen a proof that $P_{i}$ is corrupt before it terminates itself. Otherwise the termination of $P_{r}$ would be considered a deviation and an honest $P_{r}$ could be falsely detected. In the following we do not always mention explicitly that the detection sub-protocol is run as part of all protocols.

Transmission Functionality. The transmission protocol can run in two modes. In cheap mode $\mathcal{F}_{\text {TRAnSmit }}$ is implemented as follows. 
1. On input (transmit, $m i d, m)$ party $P_{s(m i d)}$ signs $(m i d, m)$ to obtain signature $\sigma_{s}$ and sends $\left(\right.$ mid, $\left.m, \sigma_{s}\right)$ to $P_{r(m i d)}$.

2. On input (transmit, mid) party $P_{r(m i d)}$ waits for one round and then expects a message $\left(m i d, m, \sigma_{s}\right)$ from $P_{s(m i d)}$, where $\sigma_{s}$ is a valid signature from $P_{s}$ on $(\mathrm{mid}, \mathrm{m})$. If it receives it, it outputs $(\mathrm{mid}, \mathrm{m})$.

3. On input (reveal, mid, $i$ ) party $P_{j}$, if it at some point output (mid, $m$ ), sends $\left(m i d, m, \sigma_{s}\right)$ to $P_{i}$, which outputs $(m i d, m)$ if $\sigma_{s}$ is valid.

It is easy to check that this is a UC secure implementation under the following restrictions:

Synchronized input from honest parties: If some honest party receives input (transmit, mid), then all honest parties $P_{i} \neq P_{s(\text { mid })}$ receives the same input (transmit, $m i d$ ). Furthermore, if $P_{s(\text { mid })}$ is honest, it receives input (transmit, mid, $m$ ) for some $m$.

Signatures: Even corrupted $P_{s}$ send along the signatures $\sigma_{s}$.

The restriction synchronized input from honest can be enforced by the way the ideal functionality is used by an outer protocol, i.e., by ensuring that the honest parties agree on which message identifiers are used for which message in which rounds. This is the case for the way we use $\mathcal{F}_{\text {TRAnsmit }}$. The restriction signatures is unreasonable, and we show how to get rid of it below. We need the rule Do not commit corrupt to corrupt in $\mathcal{F}_{\text {TRANSMIT }}$ as we cannot prevent a corrupt $P_{s}$ from providing a corrupt $P_{i}$ with signatures on arbitrary messages, i.e., we cannot commit the corrupted parties to what they have sent among themselves.

As mentioned, the above implementation only works if all senders honestly send the needed signatures. If at some point some $P_{r}$ does not receive a valid signature from $P_{s}$, it publicly accuses $P_{s}$ of being corrupted and the parties switch to the below expensive mode for transmissions from $P_{s}$ to $P_{r}$.

1. On input (transmit, $m i d, m)$ party $P_{s(m i d)}$ signs $($ mid, $m$ ) to obtain signature $\sigma_{s}$ and sends $\left(\right.$ mid, $\left.m, \sigma_{s}\right)$ to all $P_{i} \neq P_{s(m i d)}$.

2. On input (transmit, $m i d$ ) parties $P_{i} \neq P_{s(m i d)}$ wait for one round and then expects a message $\left(m i d, m, \sigma_{s}\right)$ from $P_{r(m i d)}$, where $\sigma_{s}$ is a valid signature of $P_{s(m i d)}$ on $(m i d, m)$. If $P_{i}$ receives it, it sends $\left(m i d, m, \sigma_{s}\right)$ to $P_{r(m i d)}$. Otherwise, it sends a signature $\gamma_{i}$ on (corrupt, $i$ ) to all parties.

3. On input (transmit, $m i d$ ) party $P_{r(m i d)}$ waits for two rounds and then expects a message $\left(\mathrm{mid}, \mathrm{m}, \sigma_{s}\right)$ from each $P_{i}$, where $\sigma_{s}$ is a valid signature of $P_{s(m i d)}$ on $(m i d, m)$. If it arrives from some $P_{i}$, then $P_{r}$ outputs $($ mid, $m$ ).

Note that now each round of communication on $\mathcal{F}_{\text {TRAnSmit }}$ takes two rounds on the underlying network. Between two parties where there have been no accusations, messages are sent as before (Step 1 in the above protocol) and the extra round is used for silence - it is necessary that also non-accusing parties use two rounds to not lose synchronization.

If $P_{s}$ sends a valid signature to just one honest party, then $P_{r}$ gets its signature and can proceed as in optimistic mode. If $P_{s}$ does not send a valid signature to any honest party, then all $n-t$ honest $P_{i}$ send $\gamma_{i}$ to all parties and hence all 
honest parties output (corrupt, $s$ ) in the following round, meaning that $P_{s}$ was detected. Using these observations it can easily be shown that the above protocol is a UC implementation of $\mathcal{F}_{\text {TRANSmit }}$ against covert adversaries with deterrence factor 1 . Note that it is not a problem that we send $m$ in cleartext through all parties, as an accusation of $P_{s}$ by $P_{r}$ means that $P_{s}$ or $P_{r}$ is corrupt, and hence $m$ need not be kept secret.

We skipped the details of how the accusations are handled. We could in principle handle accusations by using one round of broadcast after each round of communication to check if any party wants to make an accusation. After broadcasting the accusations, the appropriate parties can then switch to expensive model. To avoid using a Byzantine agreement primitive in each round, we use a slightly more involved, but much cheaper technique which communicates less than $n^{2}$ bits in each round and which only uses a BA primitive when there are actually some accusations to be dealt with. The details are given in the next section.

In cheap mode, using $\mathcal{F}_{\text {TRANSmit }}$ adds an overhead $N \kappa$ bits compared to plain transmission, where $\kappa$ is the length of a signature and $N$ is the number of messages sent. In expensive mode this overhead is a factor $n$ larger.

Cheap Exception Handling. Consider a protocol consisting of two protocols $\pi_{\text {MAIN }}$ and $\pi_{\text {EXCEPT }}$, both for the authenticated, synchronous point-to-point model. Initially the parties run $\pi_{\text {MAIN }}$. The goal is to allow any party to raise a flag, which stops $\pi_{\text {MAIN }}$ and starts $\pi_{\text {EXCEPT }}$. With some details left out for now, this is handled as follows.

- If a party $P_{i}$ wants to stop the main protocol, it sends (stop) to all parties and stops the execution of $\pi_{\text {MAIN }}$. It records the round $R_{i}$ in which it stopped running $\pi_{\text {MAIN }}$.

- If a party $P_{i}$ receives (stop) from any party while running $\pi_{\text {MAIN }}$, it sends (stop) to all parties and stops the execution of $\pi_{\text {MAIN }}$. It records the round $R_{i}$ in which it stopped running $\pi_{\text {MAIN }}$.

- After all parties stopped they resynchronize and then run $\pi_{\text {EХсЕРт }}$.

- After having run $\pi_{\text {EXCEPT }}$, the parties agree on a round $C$ of $\pi_{\text {MAIN }}$ which was executed completely, i.e., $R_{i}>C$ for all honest $P_{i}$, and then they rerun from round $C+1$. If a party $P_{r}$ already received a message from $P_{s}$ for one of the rounds that are now rerun, then $P_{r}$ ignores any new message sent by $P_{s}$ for that round. This is to avoid that corrupted parties can change their mind on what they sent in a previous round.

The resynchronization is needed as honest parties might stop in different rounds - though at most with a staggering of one round.

The resynchronization uses a sub-protocol where the input of $P_{i}$ is the round $R_{i}$ in which it stopped. The output is some common $R$ such that it is guaranteed that $R_{i}=R$ for some honest $P_{i}$, i.e., at least one honest party stopped in round $R$. Since the honest parties stop within one round of each other, it follows that all honest parties stopped in round $R-1, R$ or $R+1$. In particular, no honest 
party stopped in round $R-2$. The parties can therefore safely set $C=R-2$, i.e., rerun from round $R-1$.

The protocol used to agree on the round $R$ proceeds as follows:

1. Each $P_{i}$ has input $R_{i} \in \mathbb{N}$ and it is guaranteed that $\left|R_{i}-R_{j}\right| \leq 1$ for all honest $P_{i}$ and $P_{j}$.

2. Let $r_{i}=R_{i} \bmod 4$ and make 4 calls to the BA functionality - name the calls $B A_{0}, B A_{1}, B A_{2}$ and $B A_{3}$. The input to $B A_{c}$ is 1 if $c=r_{i}$ or $c=r_{i}-1 \bmod 4$ and the input to $B A_{c}$ is 0 if $c=r_{i}+1 \bmod 4$ or $c=r_{i}+2 \bmod 4$.

3. Let $o_{c} \in\{0,1\}$ for $c=0,1,2,3$ denote the outcome of $B A_{c}$. Now $P_{i}$ finds the largest $R \in\left\{R_{i}-1, R_{i}, R_{i}+1\right\}$ for which $o_{R} \bmod 4=1$ and outputs $R$.

It is fairly straight forward to see that the honest parties output the same $R$ and that $R$ was always the input of some honest party. Look at two cases.

- If there exists $\rho$ such that $R_{i}=\rho$ for all honest $P_{i}$, then all honest parties input the same to the BA functionalities, and then trivially $o_{\rho-1} \bmod 4=1$, $o_{\rho \bmod 4}=1, o_{\rho+1} \bmod 4=0$ and $o_{\rho+2 \bmod 4}=0$. Consequently, at honest parties outputs $R=\rho$.

- If there exists $\rho$ such that $R_{i}=\rho$ for some honest $P_{i}$ and $R_{j}=\rho+1$ for some honest $P_{j}$, then $R_{k} \in\{\rho, \rho+1\}$ for all honest $P_{k}$, and thus all honest $P_{k}$ input 1 to $B A_{\rho \bmod 4}$, and so $o_{\rho \bmod 4}=1$. Furthermore, all honest parties input 0 to $B A_{\rho+2 \bmod 4}$, so $o_{\rho+2} \bmod 4=0$. It follows that all honest parties output $R=\rho$ if $o_{\rho+1} \bmod 4=0$ and that all honest parties output $R=\rho+1$ if $o_{\rho+1} \bmod 4=1$. Both outputs are valid.

The above protocol is an improved version of a protocol by Bar-Noy et al. [3], which in turn uses techniques from Berman et al. [7]). The protocol in [3] uses $\log (B)$ calls to the BA functionality, where $B$ is an upper bound on the input of the parties. We use just 4 .

Note that at the point where the four BAs are run, the honest parties might still be desynchronized by one round. We handle this using a technique from [16] which simulates each round in the BA protocols by three synchronous rounds in the authenticated channel model.

Commitment Functionality. The protocol uses a one-round UC commitment scheme with a constant overhead (commit to $\kappa$ bits using $\mathcal{O}(\kappa)$ bits), which can be realized with static security in the PKI model [4] given any mixed commitment scheme 11] with a constant overhead. Concretely we can instantiate such a scheme under Paillier's DCR assumption. Note that opposed to Barak et al. [4] we do not need a setup assumption: We assume honest majority and can thus, once and for all, use an active secure MPC to generate the needed setup [14]. The protocol also uses an error-correcting code (ECC) for $n$ parties which allows to compute the message from any $n-t$ correct shares.

If one is willing to use the random oracle model, UC commitment can instead be done by calling the oracle on input the message to commit to, followed by some randomness. In practice, this translates to a very efficient solution based on a hash function. 
The protocol proceeds as follows.

1. On input (commit, cid, $m), P_{s(c i d)}$ computes an ECC $\left(m_{1}, \ldots, m_{n}\right)$ of $m$. The sender then computes $c_{i} \leftarrow \operatorname{commit}_{p k_{i}}\left(m_{i}\right)$ and sends $c_{i}$ to $P_{i}$ via $\mathcal{F}_{\text {TRAnsmit }}$.

2. On input (reveal, cid, $r), P_{i}$ opens each $c_{i}$ to $P_{i}$. The opening is sent via $\mathcal{F}_{\text {TRAnsmit }}$. If any $P_{i}$ receives an invalid opening, it transfers $c_{i}$ and $m_{i}$ to all parties and $P_{s}$ is detected as a cheater. Otherwise, $P_{i}$ transfers $c_{i}$ and the opening to $P_{r}$.

3. Then $P_{r}$ collects validly opened $c_{i}$. Let $I$ be the index of these and let $m_{i}$ be the opening of $c_{i}$ for $i \in C$. If $|I|<n-t$, then $P_{r}$ waits for one round and terminates 3 If $\left(m_{i}\right)_{i \in I}$ is not consistent with a codeword in the ECC, then $P_{r}$ transfers $\left(c_{i}\right)_{i \in I}$ and the valid openings to the other parties which detect $P_{s}$ as corrupted. Otherwise, $P_{r}$ uses $\left(m_{i}\right)_{i \in I}$ to determine $m$ and outputs $(c i d, m)$.

Assuming that a commitment to $\ell$ bits have bit-length $\mathcal{O}(\max (\kappa, \ell))$, where $\kappa$ is the security parameter, the complexity of a commitment to $\ell$ bits followed by an opening is $\mathcal{O}(n \max (\kappa, \ell / n))=\mathcal{O}(n(\kappa+\ell / n))=\mathcal{O}(\ell+n \kappa)$. This is assuming that there are no active corruptions, such that $\mathcal{F}_{\text {TRANSmit }}$ has constant overhead.

Flip Functionality. To implement $\mathcal{F}_{\text {FLIP }}^{B}$ the parties proceed as follows.

1. On input (flip), all $P_{i}$ commit to a uniformly random $k_{i} \in\{0, \ldots, B-1\}$.

2 . In the next round all $P_{i}$ reveal $k_{i}$ to all parties.

3. All parties output $k=\sum_{i=1}^{n} k_{i} \bmod B$.

Under the condition that the protocol is used by the honest parties in a way that guarantees that they input (flip) in the same round, the argument that the protocol implements the functionality against a covert adversary (with deterrence 1) is straight forward.

Input Functionality. The input functionality can be implemented using a VSS with a multiplication protocol active secure against $t<n / 2$ corruptions. The VSS should have the property that it is possible to verifiable reconstruct the secret and the share of all parties given the shares of the honest partiesstandard bivariate sharing has this property. We sketch the protocol.

1. Each $P_{i}$ deals a VSS $\llbracket x_{i} \rrbracket$ of its input $x_{i}$.

2. The parties use standard techniques to compute a VSS $\llbracket d \rrbracket$ of a uniformly random $d \in \in_{\mathrm{R}}\{0,1\} \subset K$.

3. For each input $\llbracket x_{i} \rrbracket$ the parties use an actively secure multiplication protocol to compute $\llbracket x^{(i, 0)} \rrbracket=\llbracket d_{i} \cdot x_{i} \rrbracket$ and $\llbracket x^{(i, 1)} \rrbracket=\llbracket\left(1-d_{i}\right) \cdot x_{i} \rrbracket$.

Each $P_{i}$ takes its output to be $\left(x_{i}^{(j, 0)}\right)_{j=1}^{n}$ and $\left(x_{i}^{(j, 1)}\right)_{j=1}^{n}$, where $x_{i}^{(j, c)}$ is its point on the polynomial used by the sharing $\llbracket x^{(j, c)} \rrbracket$. The other values of the VSS are internal to the implementation of $\mathcal{F}_{\text {INPUT }}$ and only used for the below command.

4. On input (reveal, $i, k$ ) the parties reconstruct $\llbracket d \rrbracket$ and all $\llbracket x^{i, d} \rrbracket$ towards $P_{k}$ and $P_{k}$ computes the points $x^{(j, d)}$ of $P_{j}$ in all sharings and output $\left(x_{i}^{(j, d)}\right)_{j=1}^{n}$.

${ }^{3}$ Since we assume that at most $t$ parties are corrupted, we can assume that either $P_{s}$ is detected or $P_{r}$ receives $n-t$ commitments with corresponding valid decommitments. 


\section{References}

[1] Algesheimer, J., Camenisch, J., Shoup, V.: Efficient computation modulo a shared secret with application to the generation of shared safe-prime products. In: Yung, M. (ed.) CRYPTO 2002. LNCS, vol. 2442, pp. 417-432. Springer, Heidelberg (2002)

[2] Aumann, Y., Lindell, Y.: Security against covert adversaries: Efficient protocols for realistic adversaries. In: Vadhan, S.P. (ed.) TCC 2007. LNCS, vol. 4392, pp. 137-156. Springer, Heidelberg (2007)

[3] Bar-Noy, A., Deng, X., Garay, J.A., Kameda, T.: Optimal amortized distributed consensus. Information and Computation 120(1), 93-100 (1995)

[4] Barak, B., Canetti, R., Nielsen, J.B., Pass, R.: Universally composable protocols with relaxed set-up assumptions. In: FOCS, pp. 186-195. IEEE Computer Society, Los Alamitos (2004)

[5] Beerliová-Trubíniová, Z., Hirt, M.: Perfectly-secure MPC with linear communication complexity. In: Canetti, R. (ed.) TCC 2008. LNCS, vol. 4948, pp. 213-230. Springer, Heidelberg (2008)

[6] Ben-Or, M., Goldwasser, S., Wigderson, A.: Completeness theorems for noncryptographic fault-tolerant distributed computation (extended abstract). In: STOC, pp. 1-10 (1988)

[7] Berman, P., Garay, J.A., Perry, K.J.: Optimal early stopping in distributed consensus. In: Segall, A., Zaks, S. (eds.) WDAG 1992. LNCS, vol. 647, pp. 221-237. Springer, Heidelberg (1992)

[8] Bogetoft, P., Christensen, D.L., Damgård, I., Geisler, M., Jakobsen, T., Krøigaard, M., Nielsen, J.D., Nielsen, J.B., Nielsen, K., Pagter, J., Schwartzbach, M.I., Toft, T.: Secure multiparty computation goes live. In: Dingledine, R., Golle, P. (eds.) FC 2009. LNCS, vol. 5628, pp. 325-343. Springer, Heidelberg (2009)

[9] Canetti, R.: Universally composable security: A new paradigm for cryptographic protocols. In: FOCS, pp. 136-145. IEEE, Los Alamitos (2001)

[10] Damgård, I., Nielsen, J.B.: Scalable and unconditionally secure multiparty computation. In: Menezes, A. (ed.) CRYPTO 2007. LNCS, vol. 4622, pp. 572-590. Springer, Heidelberg (2007)

[11] Damgård, I.B., Nielsen, J.B.: Perfect hiding and perfect binding universally composable commitment schemes with constant expansion factor. In: Yung, M. (ed.) CRYPTO 2002. LNCS, vol. 2442, pp. 581-596. Springer, Heidelberg (2002)

[12] Damgård, I., Ishai, Y., Krøigaard, M., Nielsen, J.B., Smith, A.: Scalable multiparty computation with nearly optimal work and resilience. In: Wagner, D. (ed.) CRYPTO 2008. LNCS, vol. 5157, pp. 241-261. Springer, Heidelberg (2008)

[13] Damgård, I., Geisler, M., Nielsen, J.B.: From passive to covert security at low cost. Cryptology ePrint Archive, Report 2009/592 (2009), http://eprint.iacr.org/

[14] Goldreich, O., Micali, S., Wigderson, A.: How to play any mental game - a completeness theorem for protocols with honest majority. In: STOC, pp. 218-229. ACM, New York (1987)

[15] Goyal, V., Mohassel, P., Smith, A.: Efficient two party and multi party computation against covert adversaries. In: Smart, N.P. (ed.) EUROCRYPT 2008. LNCS, vol. 4965, pp. 289-306. Springer, Heidelberg (2008)

[16] Lindell, Y., Lysyanskaya, A., Rabin, T.: Sequential composition of protocols without simultaneous termination. In: Proceedings of the twenty-first annual symposium on Principles of distributed computing, pp. 203-212. ACM Press, New York (2002) 